\title{
El desarrollo de las pequeñas y medianas empresas: Una formación de las aulas hacia la internacionalización
}

\author{
Volumen XIX No (1). Enero-Junio 2019. Pág. I-IV
}

ISSN: 0121-1048 IMPRESO ISSN: 2422-3220 EN LÍNEA

\author{
León-Castro, Ernesto* \\ Universidad De La Salle Bajío Av. Universidad 602 Lomas del Campestre \\ C.P. 37150 León, Guanajuato, México eleon@delasalle.edu.mx
}

\section{INTRODUCCIÓN}

En la actualidad el desarrollo de las pequeñas y medianas empresas (Pymes) en el mundo es un tema vital derivado del impacto económica y social que crean. Por ejemplo, estas aportan del 55\% al 65\% del total del Producto Interno Bruto (PIB) y generan del $60 \%$ al 70\% del empleo (Keskgn et al., 2010; Zafar y Mustafa, 2017). Particularmente, en el caso de las economías en vías de desarrollo, situación de la mayoría de los países de Latinoamérica, las Pymes conforman alrededor del 99\% de las empresas existentes en el mercado (Fjose y Grünfeld, 2010; Chege y Wang, 2020). Esta situación demuestra la importancia que tienen estas empresas dentro de los diferentes países, por lo que la formación de capacidades, habilidades y competencias para el manejo eficiente $y$ efectivo de las mismas (St-Jean y Audet, 2012; Dobina et al., 2019).

La literatura sobre competencias gerenciales/administrativas es amplia, por ejemplo, Max Weber (1971) señala las competencias rigurosas para la administración burocrática. Por otro lado, McClelland (1973) propone la evaluación de los individuos de acuerdo con criterios que contemplaran conocimientos y habilidades. Boyatzis (1982) da una definición formal de competencias señalando que es una característica subyacente en una persona que esta causalmente relacionada con un desempeño en un puesto de trabajo y organización concreta. Además, señala una diferencia entre las competencias umbrales (mínimas) y superiores (sobresalientes). Por su parte, Mole et al. (1993) generaliza las actitudes y atributos de la persona con los conocimientos adquiridos en la formación para presentar una pauta de comportamientos y habilidades cognitivas que cualquier gerente debería tener. Asimismo, Bird (1995) indica que las competencias son características fundamentales que incluyen motivación, habilidades, destrezas y conocimiento. También, Hayton y McEcoy (2006) definen a las competencias como la integración de conocimiento, habilidades y destrezas particulares con otras características

- Citar: León-Castro, Ernesto (2019). El desarrollo de las pequeñas y medianas empresas: Una formación de las aulas hacia la internacionalización. Inquietud Empresarial, XIX (1), I-IV. 
personales para tener un rendimiento positivo. Bajo esta misma perspectiva, Mamabolo et al., 2017 definen competencia como la capacidad de obtener de una manera satisfactoria o que supere los criterios de rendimiento establecidos resultados mediante una combinación de conocimientos, habilidades y otras características de la personalidad.

Dentro de la literatura se encuentran diversos estudios donde se señalan las competencias que se deben desarrollar para ser un administrador eficiente, entre ellos se encuentra el estudio realizado por Lombana et al. (2014) en Colombia, en este se identifica que es necesario tener la capacidad de desarrollar nuevas competencias, dentro de las que se destacan la capacidad de aprender y actualizarse de forma permanente, la cual permitirá trabajar en contextos internacionales y administrar de forma eficiente la infraestructura tecnológica de la empresa. Por otro lado, Wulany et al. (2019) identifican 27 competencias relacionadas por Pymes exitosas en Indonesia dentro de las que destacan el uso de tecnologías para promocionar sus productos y la proactividad para participar en diferentes cursos $y$ programas de capacitación gubernamentales. De igual manera, existen estudios relacionados con competencias específicas para ciertos sectores y actividades como son: transporte público (Rezk et al., 2018), hotelero (Bharwani y Talib, 2017), restaurantero (Wu, 2018), enfermería (Goktepe et al., 2018), recursos humanos (Ganesh, 2018), entre otros.

Basado en lo anterior, es evidente la importancia que tiene la formación para la generación de nuevas competencias en los gerentes y administradores, donde el rol de la educación juega un papel vital. En ese sentido, la docencia tradicional ha cambiado $y$ se han generado nuevas formas $y$ metodologías pedagógicas que tiene como finalidad ampliar las competencias y el nivel de dominio de estas dentro de los estudiantes. Por ejemplo, Delaney et al. (2017) presentan el proceso de implementación de educación basada en solución de problemas (problembased learning) e identifican diversos beneficios y problemas de utilizar dicha metodología. Por su parte, Jackson y Wilton (2016) discuten diferentes estrategias para mejorar las competencias de los estudiantes de administración a través de aprendizaje basado en trabajo (work-integrated learning).

Todo lo anterior busca potenciar dentro de los futuros administradores y emprendedores competencias que permitan prolongar el tiempo de vida de las empresas, ya que dentro de los países de Latinoamérica las cifras no supervivencia empresarial no son alentadoras. Dentro del primer año de operación de las empresas en Chile y Argentina sobreviven 5 de cada 10 , en Colombia 4 y en México solamente 3 , las cuales si se comparan con las de países como Bélgica, Eslovenia u Holanda donde la tasa de supervivencia es superior al $90 \%$. Esto genera una oportunidad importante de mejora, ya que, de no realizarse, la elevada tasa de mortalidad puede desincentivar a emprendedores potenciales, obstaculizando el crecimiento económico e innovación (Confecámaras, 2017).

Otro aspecto importante para el incremento de la tasa de supervivencia de las Pymes es la internacionalización. Lee et al., (2012) realizan un estudio con 1612 Pymes coreanas donde resaltan que la internacionalización no incrementa el riesgo al fracaso, sino que aumenta las posibilidades de supervivencia en el mercado de estas. De 
igual manera, diversos autores han realizado estudios donde se encuentra que un enfoque internacional aumenta el tiempo de vida y supervivencia de la empresa dentro del mercado a través del cambio constante de estrategia, la cual debe adaptarse a los nuevos mercados, clientes y tecnología de los cambios rápidos de la globalización (Geoger et al., 2019; Sui y Baum, 2014; Zahra et al., 2000; Autoio et al., 2000).

En conclusión, se observa la importancia del desarrollo eficiente y eficaz de las pequeñas y medianas empresas, ya que son estas el motor de la economía derivada de su aportación al PIB y al empleo en la mayoría de los países del mundo. Ante esto, el rol de la educación correcta de competencias dentro de la formación de los futuros administradores/gerentes/emprendedores permitirá mejorar las posibilidades de supervivencia dentro del mercado, que para el caso de Latinoamérica son bajos a comparación de otros países, principalmente los europeos. Finalmente, se destaca la importancia de la internacionalización de las empresas como un elemento que permite aumentar las posibilidades de crecimiento y vida de las empresas en los mercados globalizados y cambiantes en los que se trabaja en la actualidad.

\section{REFERENCIAS}

Autio, E., Sapienza, H. J., y Almeida, J. G. (2000). Effects of age at entry, knowledge intensity, and imitability on international growth. Academy of management journal, 43(5), 909-924.

Bharwani, S., y Talib, P. (2017). Competencies of hotel general managers: A conceptual framework. International Journal of Contemporary Hospitality Management.

Boyatzis, R. (1982). The competent manager: A model for effective performance. NewYork: John Wiley and Sons.

Chege, S. M., y Wang, D. (2020). Information technology innovation and its impact on job creation by SMEs in developing countries: an analysis of the literature review.
Technology Analysis y Strategic Management, 32(3), 256271.

Confecámaras, Red de Cámaras de Comercio de Colombia. (2017). Determinantes de la supervivencia empresarial en Colombia.

Delaney, Y., Pattinson, B., McCarthy, J., y Beecham, S. (2017). Transitioning from traditional to problem-based learning in management education: the case of a frontline manager skills development programme. Innovations in Education and Teaching International, 54(3), 214-222.

Dobina, T., Haidukevych, K., Panchenko, S., y Petrova, I. (2019). Effectiveness analysis of entrepreneurship model of development qualities of future managers. Journal of Entrepreneurship Education.

Fjose, S., Grünfeld, L. A., y SQW, C. G. (2010). Identifying SME roles and obstacles to SME growth. MENONpublication no. 14.

Ganesh, A. (2018). Competencies of HR Manager in Managing the Employee Welfare and Development in Construction Chemical Industry. Training y Development Journal, 9(1), 10-17.

George, A. D., Mathew, L. S., y Chandramohan, G. (2019). Internationalization of SMEs: A Darwinian Perspective. In Transnational Entrepreneurship (pp. 363-378). Springer, Singapore.

Goktepe, N., Turkmen, E., Badir, A., Hayta, O., Yakar, H. K., y Buyukgonenc, L. A. (2018). Development of managerial competencies for first-level nurse managers in Turkey. International Journal of Caring Sciences, 11(2), 10961102.

Jackson, D., y Wilton, N. (2016). Developing career management competencies among undergraduates and the role of work-integrated learning. Teaching in Higher Education, 21(3), 266-286.

Keskġn, H., Gentürk, C., Sungur, O., y K, H. M. (2010). The importance of SMEs in developing economies. In 2nd International Symposium on Sustainable Development (pp. 183-192).

Lee, H., Kelley, D., Lee, J., y Lee, S. (2012). SME survival: The impact of internationalization, technology resources, and alliances. Journal of small business management, 50(1), 119.

Lombana, J., Cabeza, L., Castrillón, J., y Zapata, Á. (2014). Formación en competencias gerenciales. Una mirada desde los fundamentos filosóficos de la administración. Estudios Gerenciales, 30(132), 301-313.

Mamabolo, M. A., Kerrin, M., y Kele, T. (2017). Entrepreneurship management skills requirements in an emerging economy: A South African outlook. The Southern African Journal of Entrepreneurship and Small Business Management, 9(1), 1-10. 
McClelland, D. (1973). Testing for competence rather than for Intelligence. American Psychologist, 28(1), 1-14.

Mole, V., Dawson, S., Winstanley, D. y Sherval, J. (1993). Researching managerial competences. British Academy of Management Annual Conference, Milton Keynes, September.

Rezk, S., Whited, G., y Hanna, A. (2018). Quantitative Assessment of Project Manager Competencies for Wisconsin Department of Transportation. In Construction Research Congress 2018 (pp. 702-711).

St-Jean, E., y Audet, J. (2012). The role of mentoring in the learning development of the novice entrepreneur. International Entrepreneurship and Management Journal, 8(1), 119-140.

Sui, S., y Baum, M. (2014). Internationalization strategy, firm resources and the survival of SMEs in the export market. Journal of International Business Studies, 45(7), 821-841.

Weber, M. (1971). Économie et société. París: Plon. De la versión alemana Wirtschaftund Gesellschaft (1921)

$\mathrm{Wu}, \mathrm{M} . \mathrm{H}$. (2018). Comparative analysis of perceived employable competency by restaurant managers of taiwan. International Journal of Organizational Innovation (Online), 11(1), 1-10.

Wulani, F., Lindawati, T., y Putro, A. J. (2019). The effectiveness of SMEa: Exploratory study of SME owners' competency in Indonesia. Journal of International Business Research (JIBR), 18(1), 1-8.

Zafar, A., y Mustafa, S. (2017). SMEs and its role in economic and socio-economic development of Pakistan. International Journal of Academic Research in Accounting, Finance and Management Sciences, 6(4).

Zahra, S. A., Ireland, R. D., y Hitt, M. A. (2000). International expansion by new venture firms: International diversity, mode of market entry, technological learning, and performance. Academy of Management journal, 43(5), 925-950. 